\title{
Assessing changes in cervical epidural pressure during biportal endoscopic lumbar discectomy
}

\author{
Taewook Kang, MD, Si Young Park, MD, PhD, Soon Hyuck Lee, MD, PhD, \\ Jong Hoon Park, MD, PhD, and Seung Woo Suh, MD, PhD \\ Department of Orthopaedics, Korea University College of Medicine, Anam Hospital, Seoul, Korea
}

\begin{abstract}
OBJECTIVE Biportal endoscopic spinal surgery has been performed for several years, and its effectiveness is well known; however, no studies on its safety, specifically intracranial pressure, have been conducted to date. The authors sought to evaluate the effect of biportal endoscopic lumbar discectomy on intracranial pressure by monitoring cervical epidural pressure (CEP) changes throughout the procedure.
\end{abstract}

METHODS Twenty patients undergoing single-level biportal endoscopic lumbar discectomy were enrolled in this study. CEPs were monitored throughout the procedure, consisting of phase 1, establishing the surgical portal and working space; phase 2, performing decompression and discectomy; and phase 3, turning off the fluid irrigation system. After discectomy was completed, the authors evaluated changes in CEP as the irrigation pressure increased serially by adding phase 4 , increasing irrigation pressure with outflow open; and phase 5 , increasing irrigation pressure with outflow closed.

RESULTS The mean baseline CEP was measured as $16.65 \mathrm{~mm} \mathrm{Hg}$. In phase 1, the mean CEP was $17.3 \mathrm{~mm} \mathrm{Hg}$, which was not significantly different from the baseline CEP. In phase 2, the mean CEP abruptly increased up to $35.1 \mathrm{~mm} \mathrm{Hg}$ when the epidural space was first connected with the working space, followed by stabilization of the CEP at $31.65 \mathrm{~mm}$ $\mathrm{Hg}$. In phase 4, the CEP increased as the inflow pressure increased, showing a linear correlation, but not in phase 5 . No patients experienced neurological complications.

CONCLUSIONS It is important to ensure that irrigation fluid is not stagnant and is maintained continuously. More attention must be paid to keeping pressures low when opening the epidural space.

https://thejns.org/doi/abs/10.3171/2020.6.SPINE20586

KEYWORDS lumbar disc herniation; biportal endoscopic lumbar discectomy; cervical epidural pressure; intracranial pressure

$\mathrm{T}$ HROUGHOUT the last century, surgical treatment of lumbar disc herniation has evolved significantly. Currently, microendoscopic discectomy is considered the standard intervention and the most common procedure performed for lumbar disc herniation. Endoscopic lumbar discectomy is known to have many advantages over conventional open lumbar discectomy. ${ }^{1,2}$ It is considered safe and effective, and associated clinical results are similar to those of the microendoscopic technique, with the advantages of shorter operation times, potential for rapid rehabilitation, and reduced postoperative pain levels. ${ }^{3}$

During endoscopic procedures, a potential working cavity needs to be created for better visualization and the application of the endoscopic instruments. For this reason, it is necessary to conduct continuous saline irrigation in the epidural space. However, increased pressure can lead to complications. ${ }^{4}$ Percutaneous endoscopic lumbar discectomy (PELD) employs a uniportal endoscope that involves multiple channels for irrigation, visualization, instrumentation, and a light source. However, this single portal limits the movement of the different instruments and obscures visualization of the operating field. Another important limitation is that it hampers the outflow of continuous inflowing irrigation fluid, which can increase the intracranial pressure (ICP) and lead to complications. It has been reported that some patients complained of severe posterior neck pain and developed seizures during PELD. ${ }^{4,5}$ These phenomena are thought to be associated with the increased cervical epidural pressure (CEP) and ICP caused by the use of irrigation fluid. ${ }^{4}$

Biportal endoscopic lumbar discectomy (BELD) in-

ABBREVIATIONS BELD = biportal endoscopic lumbar discectomy; CEP = cervical epidural pressure; ICP = intracranial pressure; PELD = percutaneous endoscopic lumbar discectomy.

SUBMITTED April 13, 2020. ACCEPTED June 18, 2020.

INCLUDE WHEN CITING Published online October 30, 2020; DOI: 10.3171/2020.6.SPINE20586. 
volves two separate portals for viewing and working. With this approach, irrigation fluid inflow through the cranial viewing portal and outflow through the caudal working portal facilitate continuous irrigation. Continuous irrigation during the procedure washes away bone debris and potentially invading microorganisms to prevent infection. ${ }^{6}$ Also, it is thought to avoid increasing ICP.

To the best of our knowledge, this is the first study reporting cervical pressure measurements during BELD. In this study, we measured the CEP continuously to investigate changes in CEP during the course of BELD.

\section{Methods \\ Study Design}

We hypothesized that any changes in CEP during BELD would not be significant in conjunction with constant outflow. To explore this, we conducted a prospective observational study of 20 patients scheduled for singlelevel BELD at our institution between May 1, 2019, and July 31, 2019.

This study was approved by the institutional review board of our institution and registered with the Clinical Research Information Service, conforming to the WHO International Clinical Trials Registry Platform (registration no. KCT0004075). All participants were given extensive written and verbal information about the trial, including its potential benefits and risks, before providing written informed consent.

The study inclusion criteria were as follows: 1) neurological signs including radiculopathy, sensory changes, and motor weakness due to disc herniation at a single level; 2) symptoms corresponding with preoperative MRI findings; 3) unsuccessful conservative treatment for at least 4 weeks; 4) no past history of spinal surgery; and 5) an age of 18-65 years. The exclusion criteria were as follows: 1) history of increased ICP or conditions that could provoke changes in the ICP (e.g., cerebrovascular events, hemorrhage, and intracranial space-occupying tumors); 2) cervical radicular pain caused by disc herniation or spinal stenosis; 3) central stenosis ( $\leq 10 \mathrm{~mm}$ ) or lateral recess stenosis $(\leq 3 \mathrm{~mm}$ ) confirmed by MRI; 4) segmental instability confirmed by dynamic radiography; 5) infection; 6) allergy to contrast media; and 7) current bleeding disorders or having received an anticoagulant within the last 10 days.

All patients underwent general anesthesia with intravenous induction (fentanyl, propofol, and vecuronium or rocuronium) and inhalational maintenance with isoflurane. The esophageal temperature was maintained above $36^{\circ} \mathrm{C}$, and the mean systolic blood pressure was maintained under $120 \mathrm{~mm} \mathrm{Hg}$. Measures of blood pressure were taken at induction and measured every 30 minutes throughout the procedure.

\section{Cervical Epidural Pressure Measurement}

With fluoroscopic guidance, a standard 18-gauge Tuohy needle was inserted at the vertebral level of C7-T1. The loss of resistance technique with a midline approach was adopted for insertion of the needle into the epidural space. A 22-gauge epidural catheter (FlexTip Plus Epi- dural Catheter) was advanced via the Tuohy needle in a cephalad direction to the C6-7 level. The correct catheter placement was confirmed using contrast fluoroscopy in the cervical anteroposterior and lateral views. The catheter was attached to a pressure transducer (AutoTransducer, Ace Medical) and the patient monitoring system, and the zero level was set at the height of the external auditory meatus.

CEP was monitored throughout the procedure, consisting of the following initial stages: phase 1, establishing the surgical portal and working space; phase 2, performing decompression and discectomy; and phase 3 , turning off the fluid irrigation system. After the discectomy was completed, we evaluated further possible changes in CEP as the irrigation pressure increased $10 \mathrm{~mm} \mathrm{Hg} / \mathrm{min}$ serially from $30 \mathrm{~mm} \mathrm{Hg}$ to $80 \mathrm{~mm} \mathrm{Hg}$ during two additional stages: phase 4 , increasing the irrigation pressure with the outflow open; and phase 5, increasing the irrigation pressure with the outflow closed.

\section{Surgical Procedure}

All patients underwent surgery in a prone position under general anesthesia by one experienced surgeon. Two separate oblique $1-\mathrm{cm}$-long skin incisions were made above and below the disc space, respectively. The viewing portal was established cranially, and a $0^{\circ}$ arthroscope was inserted. A saline irrigation pump was connected to the viewing portal and set to a pressure of $30 \mathrm{~mm} \mathrm{Hg}$. Continuous flow of saline perfusion was essential to prevent excessive elevation of the epidural pressure, which can provoke an elevation in the ICP (Fig. 1). The working portal was made caudally, and conventional spinal surgical instruments and arthroscopic instruments were inserted through this portal. Hemilaminectomy was performed using a burr and a Kerrison rongeur until the upper edge of the deep portion of the ligamentum flavum was exposed. The ligamentum flavum was carefully dissected and completely resected. An incision was made on the disc and pituitary forceps, and curettes were employed to complete a discectomy and remove fragments of the herniated discs. The procedure was finished after confirming the decompression status and freely moving the exiting nerve root with a ball-tip probe. A Hemovac drain was placed in all patients through the working portal to prevent hematoma formation.

We used an irrigation pump (Storz) whose pressure was adjusted to fall between 30 and $60 \mathrm{~mm} \mathrm{Hg}$ for bleeding control. If severe bleeding obscured the endoscopic visualization, increasing the irrigation pressure can be adopted as a maneuver to control it. Elevating the irrigation pressure overcomes the pressure of the venous bleeding, allowing for visualization and cauterization of the bleeding vessel. The pressure is lowered back to the initial pressure as soon as the bleeding is controlled.

\section{Statistical Analysis}

The Friedman ANOVA test was used to compare the nonnormally distributed CEPs of different phases. A post hoc analysis was performed with the Wilcoxon signedrank test, and Bonferroni correction was used to adjust 


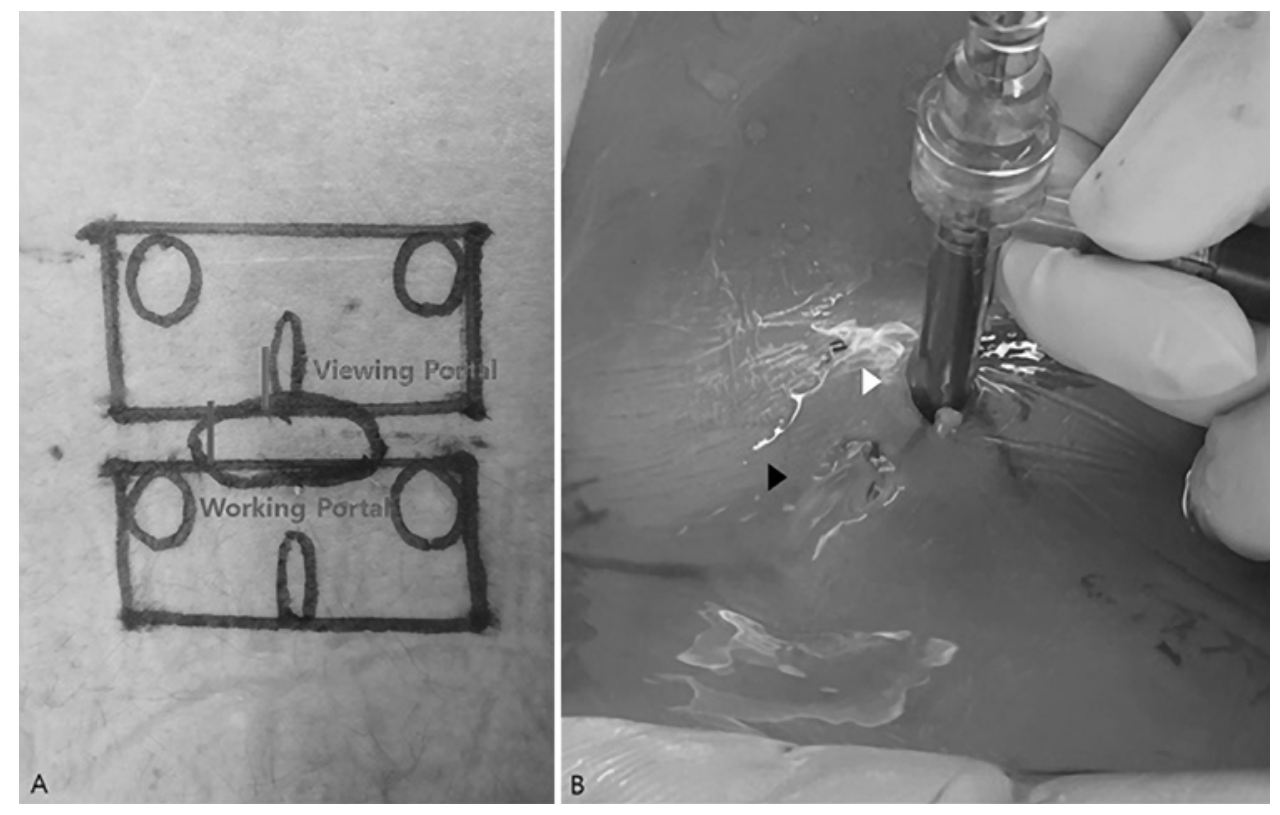

FIG. 1. A: Portal placement for BELD. B: Constant outflow through the working portal. The white arrowhead indicates the viewing portal and the black arrowhead the working portal. Figure is available in color online only.

for multiple comparisons. The level of significance was set at $\mathrm{p}<0.05$. All statistical analyses were performed using IBM SPSS version 20.0 (IBM Corp.).

\section{Results}

\section{Demographic Data}

The general characteristics and intraoperative data of the patients are presented in Table 1. All patients were followed up for at least 6 months after surgery. There were 11 men and 9 women, and the mean age $( \pm$ SD) was $43.75 \pm$ 13.23 years. The operative levels were L3-4 in 1 patient, L4-5 in 11 patients, and L5-S1 in 8 patients. The total operation time was $42.5 \pm 9.93$ minutes. The mean systolic blood pressure was $105.1 \pm 8.3 \mathrm{~mm} \mathrm{Hg}$. There were no dura tears or cases of CSF leakage. At the time of the last follow-up, none of the patients had developed neurological complications.

TABLE 1. Demographic data of patients undergoing BELD

\begin{tabular}{cc}
\hline & Value \\
\hline Mean age, yrs & $43.75 \pm 13.23$ \\
\hline M/F sex & $11: 9$ \\
\hline Level & 1 \\
\hline L3-4 & 11 \\
\hline L4-5 & 8 \\
\hline L5-S1 & $42.5 \pm 9.93$ \\
\hline Mean operation time, mins & $105.1 \pm 8.3$ \\
\hline Mean systolic blood pressure, $\mathrm{mm} \mathrm{Hg}$ & 0 \\
\hline No. of complications &
\end{tabular}

Values represent the number of patients unless stated otherwise. Mean values are presented as the mean \pm SD.

\section{Cervical Epidural Pressure Measurement}

The observed changes in CEP during surgery are shown in Table 2 and Fig. 2. CEPs at baseline and phases 1, 2, and 3 were significantly different $(\mathrm{p}<0.001)$. The baseline stabilized CEP was $16.65 \pm 9.1 \mathrm{~mm} \mathrm{Hg}$.

\section{Phase 1: Establishing the Surgical Portal and Working Space}

The mean CEP was $17.3 \pm 8.62 \mathrm{~mm} \mathrm{Hg}$ during phase 1 and was not significantly different from the baseline CEP $(\mathrm{p}=0.24)$. The maximum CEP in phase 1 was $17.55 \pm$ $8.71 \mathrm{~mm} \mathrm{Hg}$, which was not significantly different from the mean CEP in phase $1(\mathrm{p}=0.075)$.

\section{Phase 2: Performing Decompression and Discectomy}

An abrupt increase in CEP up to $35.1 \pm 11.44 \mathrm{~mm} \mathrm{Hg}$ was observed when the epidural space was connected with the outer working space. The maximum CEP in phase 2 was $45.2 \pm 21.92 \mathrm{~mm} \mathrm{Hg}$ and was recorded a mean of 23.65 \pm 18.91 seconds after opening the epidural space. Then the CEP gradually decreased and stabilized at $31.65 \pm 9.38$

TABLE 2. Changes in CEP at different phases

\begin{tabular}{cc}
\hline & Mean \pm SD $(\mathrm{mm} \mathrm{Hg})$ \\
\hline Baseline CEP & $16.65 \pm 9.1$ \\
\hline Phase 1 & $17.3 \pm 8.62$ \\
\hline Maximum & $17.55 \pm 8.71$ \\
\hline Phase 2 & $35.1 \pm 11.44$ \\
\hline Maximum & $45.2 \pm 21.92$ \\
\hline Maintenance & $31.65 \pm 9.38$ \\
\hline Phase 3 & $21.4 \pm 8.34$ \\
\hline
\end{tabular}




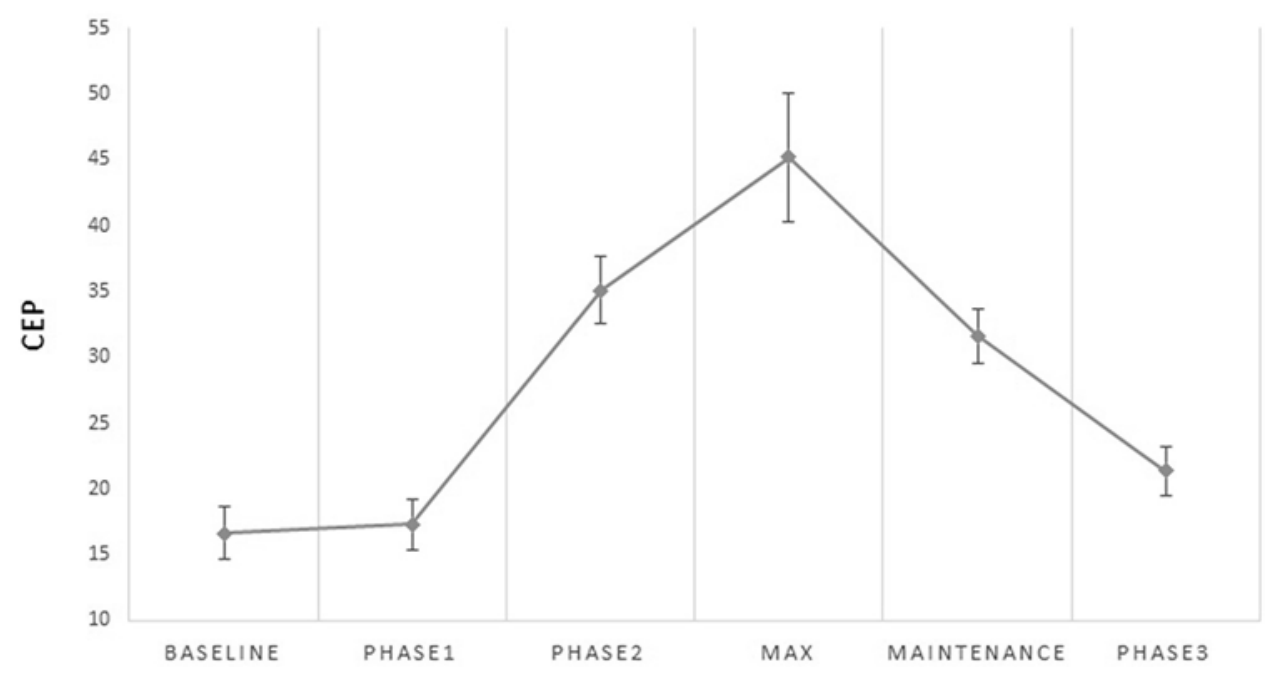

FIG. 2. Mean CEP (in $\mathrm{mm} \mathrm{Hg}$ ) changes in phases 1 through 3. Max = maximum. Figure is available in color online only.

$\mathrm{mm} \mathrm{Hg}$ after $64.15 \pm 15.89$ seconds, remaining significantly increased relative to the baseline CEP $(\mathrm{p}<0.001)$. There was no increase in CEP as the operation time continued.

\section{Phase 3: Turning Off the Fluid Irrigation System}

After halting irrigation, the elevated CEP was recovered and stabilized at $21.4 \pm 8.34 \mathrm{~mm} \mathrm{Hg}$ in $36.05 \pm 14.33$ seconds. This was significantly different from the baseline CEP $(p<0.001)$.

\section{Phase 4: Increasing the Pump Pressure With the Outflow Open}

The CEP increased until the inflow pressure increased to $50 \mathrm{~mm} \mathrm{Hg}$ and then plateaued (Fig. 3). As the pressure increased by $10 \mathrm{~mm} \mathrm{Hg} / \mathrm{min}$ up to $50 \mathrm{~mm} \mathrm{Hg}$, the CEP increased by $3.8 \mathrm{~mm} \mathrm{Hg}$. It took $23.4 \pm 5.14$ seconds for the CEP to stabilize when the pressure increased from 30 to $50 \mathrm{~mm} \mathrm{Hg}$. When the pressure increased from 50 to $80 \mathrm{~mm} \mathrm{Hg}$, the CEP was unchanged at $30.55 \pm 10.69$ $\mathrm{mm} \mathrm{Hg}$.

\section{Phase 5: Increasing the Pump Pressure With the Outflow Closed}

The CEP increased as the inflow pressure increased, showing a linear correlation (Fig. 4). As the pressure increased by $10 \mathrm{~mm} \mathrm{Hg} / \mathrm{min}$, the CEP increased by 9.84 $\mathrm{mm} \mathrm{Hg}$. The time taken until the CEP stabilized gradually decreased as the pressure increased; it took $23.5 \pm$ 4.93 seconds for the CEP to stabilize when the pressure increased from 30 to $40 \mathrm{~mm} \mathrm{Hg}$ but $10.05 \pm 3.5$ seconds for the CEP to stabilize when the pressure increased from 70 to $80 \mathrm{~mm} \mathrm{Hg}$.

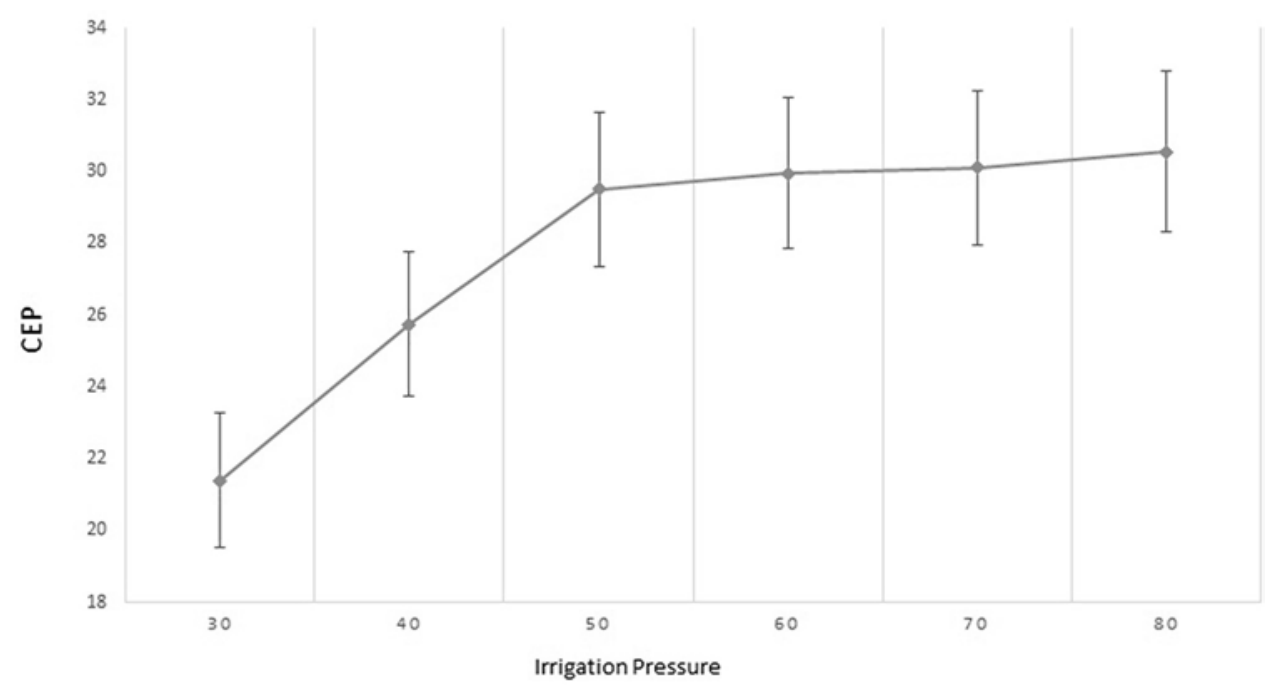

FIG. 3. Mean CEP (in $\mathrm{mm} \mathrm{Hg}$ ) changes in phase 4. Figure is available in color online only. 


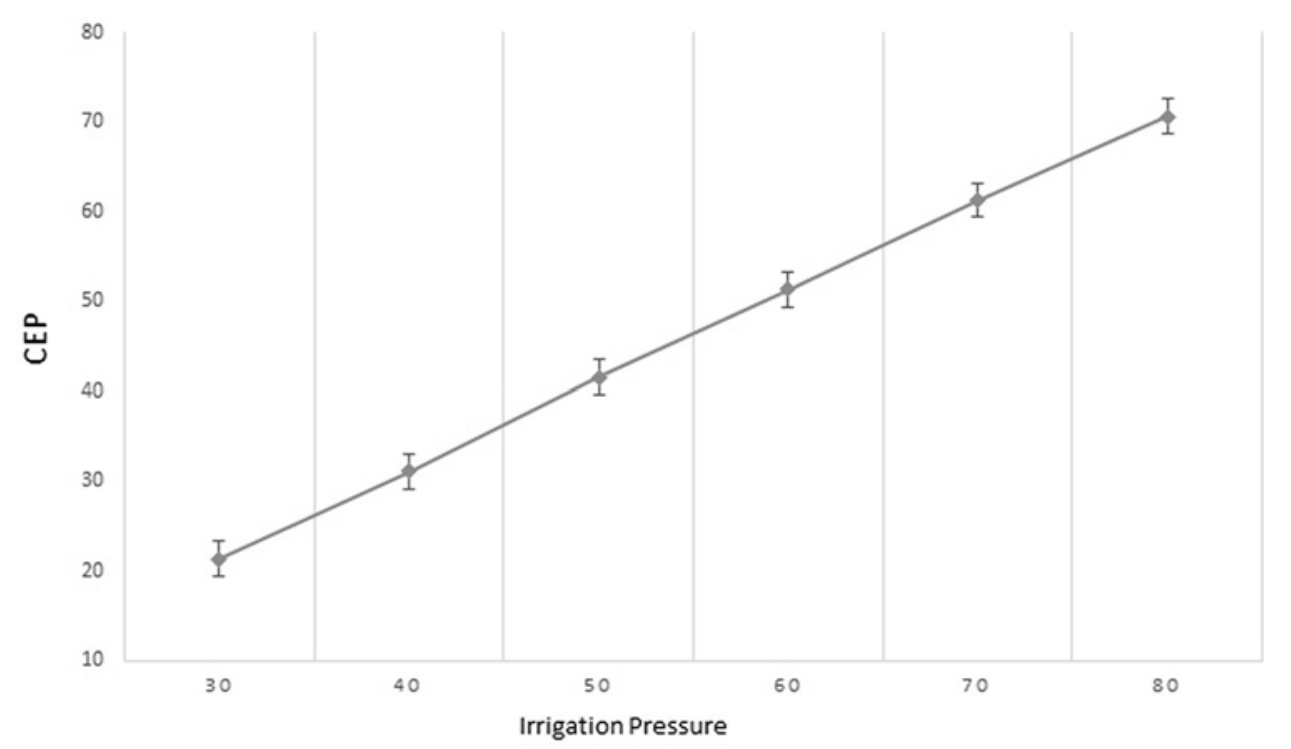

FIG. 4. Mean CEP (in $\mathrm{mm} \mathrm{Hg}$ ) changes in phase 5. Figure is available in color online only.

\section{Discussion}

The purpose of this study was to investigate changes in the CEP during the BELD and to confirm the safety of the procedure. No patients in our study experienced neurological complications. The CEP was stable and did not fluctuate before the epidural space was connected with the outer working space, but it abruptly and significantly increased once the ligamentum flavum was incised and the epidural space was exposed. Although there was a significant increase in the CEP with a closed outflow, the CEP did not increase significantly with constant outflow.

Dura injury, nerve root injury, and epidural hematoma are possible complications of endoscopic spinal surgery, but the incidence rates of these conditions are not high. However, the surgeon must remain aware of the potential for an increase in ICP during surgery, which may lead to headaches, seizures, or even death. Several studies have reported an increase in ICP during PELD. Choi et al. identified 4 cases of seizure among 16,725 patients who underwent PELD. ${ }^{5}$ Elsewhere, Joh et al. monitored the CEPs of 28 patients during PELD, including 8 who developed posterior neck pain, and high CEPs were noted when posterior neck pain occurred. ${ }^{4}$ These authors concluded that posterior neck pain is induced by the onset of high CEP correlated with continuous irrigation. Shin et al. reported a case of nonepileptic seizure after PELD under general anesthesia. ${ }^{7}$ An increase in ICP should not be ignored because it can lead to serious complications and may be fatal if the surgeon does not expect it to happen. ${ }^{8,9}$ However, none of these studies paid attention to the possible risk of increased ICP during BELD.

BELD combines the advantages of conventional open surgery and PELD. It enables surgeons to have a good and wider field of vision with high magnification. Ordinary arthroscopic and spine instruments can be used during this procedure without the need for special endoscopic instruments. A greater surgical range of motion with free move- ment, handling, and angulation of the surgical instruments and the arthroscope without crowding of the instruments is enabled as the devices are not all restricted in a single portal. By using two portals, continuous saline irrigation with clear outflow that allows the saline to flow out is possible; this approach can also control bleeding and infection.

The mechanism of ICP elevation is thought to be cranial shifting of CSF rather than the direct movement of the irrigation fluid itself. The epidural space is a potential area that spreads throughout the entire spine. Continuous irrigation of saline is required for the formation of the working space between the endoscope and the tissue to obtain better visualization and to facilitate proper operation of the instruments during the endoscopic procedure..$^{10,11}$ However, the use of a large amount of irrigation fluid and high irrigation pressure for visual accessibility is more likely to increase CEP. ${ }^{4}$ The increased intracanalicular pressure due to continuous infusion of irrigation fluid into the epidural space would compress the dural sac. As the epidural pressure elevates, the dural sac is compressed and CSF is shifted cranially. The cephalad shift of CSF will increase CEP. ${ }^{11}$ During surgery, raising the irrigation pressure is inevitable in order to obtain endoscopic visualization. The working space is the epidural space in the interlaminar approach and, when the epidural space is exposed, the irrigation fluid is infused directly into the epidural space. Bleeding from the epidural vessels will blur the endoscopic view, which leads to the use of even higher irrigation pressures. $^{4}$

The direct measurement of ICP with intracranial monitors is the gold standard but requires access to the ventricles or brain parenchyma. ${ }^{12}$ Due to its level of invasiveness with possible complications such as infection, hematoma formation, and seizure, this approach would impart an unacceptable risk in patients undergoing BELD. ${ }^{13}$ Therefore, we measured CEP as an indirect index of ICP, as CEP is known to have a linear correlation with ICP in patients without intracranial pathology. ${ }^{10,14,15}$ Hilt et al. showed a 
good correlation between baseline ICP and the increase produced by the lumbar extradural injection. ${ }^{10}$ Hamada et al. showed that the lumbar epidural pressure measurement is useful for monitoring ICP. ${ }^{15}$ CEP monitoring with cervical epidural catheterization can be easily performed with less invasiveness; ICP during PELD was measured by CEP monitoring in two other studies. ${ }^{4,5}$ To avoid measuring venous or intradural pressure, we checked that there was no CSF or blood leakage through the catheter, and correct catheter placement was confirmed by contrast injection under fluoroscopic guidance.

ICP is associated with CSF, brain tissue, and circulating cerebral blood, which coexist in a rigid space. The Monro-Kellie hypothesis states that an increase in one of the elements should be compensated for by a decrease in other elements, or it will result in the elevation of ICP. ${ }^{16}$ ICP and blood pressure interact and affect cerebral blood flow. Under normal circumstances, cerebral blood flow is made constant over a wide range of cerebral perfusion pressures by autoregulation. Cerebral blood flow is usually maintained consistently at systolic blood pressures of $50-140 \mathrm{~mm} \mathrm{Hg} .^{13}$

In phase 5, there was a significant increase in CEP as the irrigation pressure increased. The time to CEP stabilization at each pressure level decreased as the pressure increased. This is thought to be correlated with a loss of compliance. Continuous irrigation into the epidural space compresses the dura and shifts the CSF cranially. ${ }^{10}$ The elevation of ICP will be determined by the amount of CSF absorbed intracranially with the intracranial compliance of the patient. ${ }^{10,11}$ Persistent compressive pressure in the spinal canal is probably correlated with increased ICP. ${ }^{10}$ As the ICP rises, the autoregulatory mechanisms of the brain will increase the blood pressure to maintain a normal cerebral perfusion pressure. An increase in the blood pressure will cause bleeding to be more severe, which would prompt the surgeon to deliver higher irrigation pressure, leading to an even higher ICP. The surgeon should be aware of this vicious cycle and be careful to avoid it.

The most common symptoms of increased ICP are headache, papilledema, vomiting, neck stiffness, tinnitus, extremity paresthesia, low-back pain, and gait ataxia. There was no consistent relationship between the severity of symptoms and the degree of elevation of ICP, and most of the symptoms disappeared as ICP decreased. However, in some cases, the decrease in cerebral perfusion pressure due to increased ICP can lead to detrimental cerebral ischemia.

There are some precautions we need to consider when performing BELD. First, we should maintain the lowest irrigation pressure needed to obtain endoscopic visualization. CEP was maintained in a stable fashion while establishing the working space before the epidural space was opened, and the CEP abruptly increased when the ligamentum flavum was first incised. When making the working space, it is considered safe to elevate the irrigation pressure. However, after the epidural space is exposed, we must try to keep the irrigation pressure low. The higher the pressure, the more fluid that will flow into the epidural space; as a result, it will take less time for the epidural space to be filled with fluid, and the space will lose compliance.
Second, we should try to make the working portal that is the outflow portal able to handle rates of both steady flow-out and gush-out. Ensuring the irrigation fluid is not stagnant and maintained continuously enables continued clean endoscopic visualization and minimizes the possibility of neurological complications caused by elevations in CEP and ICP. There was no significant increase in CEP with constant fluid outflow. This means that significant compression on the neural component would not happen even after epidural space was exposed.

Third, the use of postoperative Hemovac drainage is essential. There was a difference seen between the baseline and final CEPs, although it was not significant. It is thought that the CEP was not elevated significantly by the autoregulation of ICP and constant outflow. However, there still might be excessive irrigation fluid in the epidural space. Hemovac placement may be helpful for facilitating drainage of excessive fluid as well as preventing hematoma formation.

Fourth, we should be very careful not to cause unnecessary bleeding. Bleeding obscures the endoscopic visualization and requires higher irrigation pressures and additional coagulation procedures to be addressed. Gentle manipulation is essential, and coagulation of the epidural vessels in advance may be helpful.

Fifth, special consideration should be taken for the patients with intracranial pathology before surgery. In patients who have intracranial pathology such as pseudotumor, hydrocephalus, or other space-occupying lesions, there will be a loss or alteration in intracranial compliance that will have an effect on the autoregulatory mechanism of ICP. Therefore, these patients may show unexpected increases in ICP. Although such conditions are not considered to be absolute contraindications, it is necessary to take great care with these patients before performing BELD.

There are several limitations to our study. First was the use of noninvasive CEP measurement because of the ethical problems related to invasive measurement, even though direct measurement of ICP is the gold standard. Second, there was a correlation between blood pressure and ICP, and we maintained hypotensive anesthesia during the surgery, but real-time changes in blood pressure were not considered. Third, because we performed BELD under general anesthesia, we could not evaluate patients' symptoms of elevated CEP during the procedure, and serious and devastating complications could occur. Fourth, it is possible that the epidural venous pressure was measured rather than the CEP. However, the epidural veins are concentrated in the anterolateral portion of the epidural space, and we tried to minimize the possibility by confirming the epidural placement of the catheter. Lastly, we could not obtain results for determining a cutoff value of the CEP, which is the limit of the elevation of ICP.

\section{Conclusions}

BELD has an advantage of allowing for continuous irrigation with a separate portal for outflow. There were no significant increases in CEP or ICP noted in the context of constant outflow. We need to ensure that the irrigation fluid is not stagnant and is maintained continuously. Fur- 
thermore, more attention must be paid to keeping pressures low when opening the epidural space.

\section{References}

1. Hanley E, Green NE, Spengler DM. An AOA critical issue. Less invasive procedures in spine surgery. J Bone Joint Surg Am. 2003;85(5):956-961.

2. Maroon JC. Current concepts in minimally invasive discectomy. Neurosurgery. 2002;51(5 Suppl):S137-S145.

3. Ruetten S, Komp M, Merk H, Godolias G. Full-endoscopic interlaminar and transforaminal lumbar discectomy versus conventional microsurgical technique: a prospective, randomized, controlled study. Spine (Phila Pa 1976). 2008;33(9): 931-939.

4. Joh JY, Choi G, Kong BJ, et al. Comparative study of neck pain in relation to increase of cervical epidural pressure during percutaneous endoscopic lumbar discectomy. Spine (Phila Pa 1976). 2009;34(19):2033-2038.

5. Choi G, Kang HY, Modi HN, et al. Risk of developing seizure after percutaneous endoscopic lumbar discectomy. $J$ Spinal Disord Tech. 2011;24(2):83-92.

6. Kim JE, Choi DJ, Park EJJ, et al. Biportal endoscopic spinal surgery for lumbar spinal stenosis. Asian Spine J. 2019;13(2): 334-342.

7. Shin SW, Baek SH, Choi BS, et al. Takotsubo cardiomyopathy associated with nonepileptic seizure after percutaneous endoscopic lumbar discectomy under general anesthesia. $J$ Anesth. 2010;24(3):460-463.

8. Gill JB, Heavner JE. Visual impairment following epidural fluid injections and epiduroscopy: a review. Pain Med. 2005; 6(5):367-374.

9. Ramsay M, Roberts C. Epidural injection does cause an increase in CSF pressure. Anesth Analg. 1991;73(5):668.

10. Hilt H, Gramm HJ, Link J. Changes in intracranial pressure associated with extradural anaesthesia. Br J Anaesth. 1986; 58(6):676-680.

11. Higuchi H, Adachi Y, Kazama T. Effects of epidural saline injection on cerebrospinal fluid volume and velocity waveform: a magnetic resonance imaging study. Anesthesiology. 2005;102(2):285-292.
12. Kirkman MA, Smith M. Intracranial pressure monitoring, cerebral perfusion pressure estimation, and ICP/CPP-guided therapy: a standard of care or optional extra after brain injury? Br J Anaesth. 2014;112(1):35-46.

13. Dunn LT. Raised intracranial pressure. J Neurol Neurosurg Psychiatry. 2002;73(suppl 1):i23-i27.

14. Cardoso MM, Carvalho JC. Epidural pressures and spread of $2 \%$ lidocaine in the epidural space: influence of volume and speed of injection of the local anesthetic solution. Reg Anesth Pain Med. 1998;23(1):14-19.

15. Hamada J, Fujioka S, Ushio Y. Clinical investigation of lumbar epidural pressure. Neurosurgery. 1993;32(5):780-784.

16. Mokri B. The Monro-Kellie hypothesis: applications in CSF volume depletion. Neurology. 2001;56(12):1746-1748.

\section{Disclosures}

The authors report no conflict of interest concerning the materials or methods used in this study or the findings specified in this paper.

\section{Author Contributions}

Conception and design: SY Park, Kang, Lee. Acquisition of data: Kang. Analysis and interpretation of data: Kang. Drafting the article: Kang. Critically revising the article: SY Park, Kang, Lee. Reviewed submitted version of manuscript: all authors. Statistical analysis: Kang, Suh. Administrative/technical/material support: Kang, Lee, JH Park, Suh. Study supervision: SY Park, Lee, JH Park.

\section{Correspondence}

Si Young Park: Korea University College of Medicine, Anam Hospital, Seoul, Korea. drspine90@kumc.or.kr. 